\title{
Benign multiple sclerosis? Clinical course, long term follow up, and assessment of prognostic factors
}

\author{
S A Hawkins, G V McDonnell
}

\begin{abstract}
Objective-To establish the characteristics of patients following a benign course of multiple sclerosis and evaluate the importance of potential prognostic factors. Also, an assessment of the value of the Kurtzke EDSS as a prognostic indicator has been undertaken in patients previously determined to have benign multiple sclerosis, after 10 years of follow up.

Methods-A prevalence study in the Coleraine, Ballymena, Ballymoney, and Moyle districts of Northern Ireland used the Kurtzke expanded disability scale score (EDSS) in 259 patients with multiple sclerosis. Of these, 181 had had multiple sclerosis for $\geqslant 10$ years, 36 having benign disease $($ EDSS $\leqslant 3.0) \geqslant 10$ years after onset. Clinical and demographic details of the various patient groups, including the minimal record of disability, were compared. The 1987 study in Northern Ireland identified 33 patients with benign multiple sclerosis. Twenty eight were available for follow up in 1996 along with 42 contemporary non-benign patients.
\end{abstract}

Results-Patients with benign multiple sclerosis were predominantly women (ratio 4.1:1 $v$ 2.1:1) and younger at onset (25.8 $v$ 31.2 years). Commonest symptoms at onset were sensory and optic neuritis $(33.3 \%$ each). Patients with late onset (older than 40 years) were less likely to have a benign course, more likely to have a progressive course from onset, significantly more likely to have motor disturbance at presentation, and had a lesser female predominance. Optic neuritis was significantly more common in those with a younger age at onset. In the follow up study, patients with benign multiple sclerosis continued to have a more favourable course than non-benign counterparts but progression of disability and to the secondary progressive phase remained significant.

Conclusions-The association of female sex, early onset, and presentation with optic neuritis and sensory symptoms with a favourable course is confirmed. However, although the EDSS does provide a useful indicator of prognosis, the label "benign multiple sclerosis" is often temporary as apparently benign disease often becomes disabling.

(F Neurol Neurosurg Psychiatry 1999;67:148-152)

Received 20 October 1998

and in revised form

16 December 1998

Accepted 20 January 1999
It is clear that the course of the disease in multiple sclerosis is characterised by a wide range of rates of progression. Subclinical cases have been identified in twin studies, in which aymptomatic twins of clinically affected patients have undergone MRI. ${ }^{1}$ One postmortem study identified five unexpected cases of multiple sclerosis ${ }^{2}$ and it was suggested that subclinical multiple sclerosis may be at least as common as clinically diagnosed disease. ${ }^{3}$

The mildest form of multiple sclerosis that is clinically apparent has been labelled "benign" multiple sclerosis. This concept is widely quoted by neurologists when helping patients to come to terms with their diagnosis. The ability to predict the subsequent clinical course of multiple sclerosis, based on the initial presentation and early disability, would be invaluable, considerably adding to the accuracy and quality of prognostic information provided for patients and leading to the most appropriate selection of patients for therapeutic interventions.

The lack of agreement as to what constitutes benign disease has contributed to the widely varying estimates as to the prevalence of benign multiple sclerosis $\left(5 \%^{4}\right.$ to $\left.40 \%{ }^{5}\right)$. One of the earliest attempts to define the benign category was by McAlpine. ${ }^{6}$ The term "benign" was used to describe a group of patients who, after a follow up period of $\geqslant 10$ years, were "without restriction of activity for normal employment and domestic life but not necessarily symptomfree". Subsequent definitions have stipulated the ability to work after 20 years $^{7}$ and "slight disability" after at least 10 years, ${ }^{8}$ and more recently many have relied on the use of the Kurtzke scales and a certain minimum disease duration. ${ }^{4}{ }^{10}$ In an international survey on defining the clinical course of multiple sclerosis there was disagreement concerning the criteria for benign disease, the consensus being that patients be "fully functional" $\geqslant 15$ years from onset. ${ }^{11}$

Considering prognostic indicators, it is generally agreed that the onset of a progressive course carries a poor prognosis. ${ }^{9}{ }^{12-15}$ However, the value of this observation is limited, as transition from a relapsing-remitting to a progressive phase can only be determined after progression has already occurred and it is therefore not a predictive or prognostic criterion.

In a detailed veterans' study it was claimed that the disability status 5 years from diagnosis is predictive of the subsequent course. ${ }^{9}$ Less than $8 \%$ of those with mild disability at 5 years (Kurtzke disability status scale (DSS) score <3) were severely disabled (DSS 6-10) 10 
years after diagnosis and only $11 \%$ were severely disabled at 15 years.

For relapse rate, McAlpine suggested that a low rate is associated with benign disease ${ }^{6}$ Confavreux et al found that the mean duration between the first and second relapse was much longer in "benign" cases. ${ }^{13}$ However, the relapse rate in that study was actually greater for benign than for more malignant multiple sclerosis. Thompson et $a l^{10}$ did find an association between short first remission (less than 1 year) and an increased risk of progressive disease but others have found no correlation between relapse rate and speed of progression. ${ }^{16}{ }^{17}$ More recently, however, significant differences were shown in survival curves of disability between patients with a high or low number of attacks in the first 2 years after onset of multiple sclerosis and also between patients with a short or long interattack interval. ${ }^{18}$ This is contrary to a 25 year follow up study of an incidence cohort of over 300 patients, in which the number of relapses during the first 2 or 5 years showed no correlation with prognosis. ${ }^{19}$

The prognostic significance in multiple sclerosis of age of onset, sex, and nature of the initial symptoms have been examined. Again, until relatively recently, there had been some controversy surrounding all of these factors. Although several investigators indicated that females had a relatively favourable course, ${ }^{20-22}$ some found no influence of sex, ${ }^{10131523}$ and one group actually found that males had a more favourable course. ${ }^{12}$ Most investigators have found a worse prognosis in patients who are older (generally $>40$ years) at onset. ${ }^{12}{ }^{1423}$ Few have shown significant differences in patients younger than 40 although one group did find a negative correlation coefficient between age of onset and the percentage of patients in each age group under 40 with a benign illness. ${ }^{10}$ Several authors have found optic neuritis to be a favourable initial symptom. ${ }^{6}{ }^{14}{ }^{23}$ By comparison, motor or cerebellar symptoms at onset have been deemed unfavourable, ${ }^{69101223}$ and a polysymptomatic onset predicts a poor prognosis according to some. ${ }^{6}{ }^{12}$

The most authoritative data regarding these factors again comes from Ontario. ${ }^{18}$ There, male sex, late onset, and being seen from onset of multiple sclerosis were all associated with an adverse outcome. Optic neuritis at onset was favourable, slow onset of a motor deficit and cerebellar involvement being associated with a poor outcome. Progressive disease from onset (with or without relapses) was a negative factor prognostically. Although Runmarker and Andersen found no benefit for optic neuritis over brainstem or spinal onset in relapsingremitting multiple sclerosis, females and those with an early onset were more likely to have a benign course. ${ }^{19}$ In addition, a monoregional onset predicted a better prognosis compared with a polyregional onset.

We recently completed a population based epidemiological study of multiple sclerosis in Northern Ireland which identified a population with apparently benign disease. ${ }^{24}$ We have undertaken a close evaluation of those patients to establish the characteristics of benign multiple sclerosis and to assess if these are compatible with the findings of others. In addition, as the prevalence study incorporates an area previously studied in 1986-7 we have been able to perform a 10 year follow up of those previously considered to have benign disease. The aim of this aspect of the study is to assess the validity of the Kurtzke expanded disability scale score (EDSS) and DSS as an indicator of the subsequent course of the disease in benign multiple sclerosis and to ascertain whether patients initially considered to have benign disease continue to have a favourable course.

\section{Methods}

PATIENT GROUPS

The database for this study has been previously described. ${ }^{24}$ In brief, an epidemiological study of multiple sclerosis was conducted in the neighbouring districts of Coleraine, Ballymena, Ballymoney, and Moyle in the north east of Northern Ireland. Several sources were used for case identification and those satisfying the Poser criteria ${ }^{25}$ were accepted as prevalent cases.

On prevalence day, 1 July 1996, 288 patients were identified with definite, probable, or suspected multiple sclerosis by the Poser criteria with 259 patients undergoing an EDSS assessment. Of these, 181 had had multiple sclerosis for at least 10 years at the time of assessment. Using widely applied criteria (Kurtzke EDSS $\leqslant 3.0$ at least 10 years after onset) ${ }^{26} 2736$ patients $(19.9 \%$ ) were considered to have benign multiple sclerosis. General clinical and demographic details were established in all patients including sex, age, age at onset, disease duration, symptoms at onset, diagnostic classification, and Kurtzke functional system (FS) scores. Other components of the minimal record of disability were also applied including the incapacity status scale and environmental status scale. ${ }^{28} 29$

For the second stage of the study, the records of the previous epidemiological study in Northern Ireland were reviewed. Conducted in 1986-7, that study involved three of the areas involved in the 1996 survey-Coleraine, Ballymoney, and Moyle. This provided a cohort of patients whose subsequent clinical course with regard to disease progression could be assessed. Of the 118 definite, probable, and possible multiple sclerosis cases identified in that survey, 33 were judged to have benign disease. Although the earlier version of the Kurtzke scale was used-the DSS which measured disability on a similar scale of $0-10$, but with 1.0 steps rather than the 0.5 increments of the EDSS - the definition of benign multiple sclerosis was effectively the same (DSS $\leqslant 3.0$ at least 10 years after onset). As well as this benign group, there were a further 42 patients who had a DSS score calculated in 1987 and an EDSS assessment performed in 1996.

\section{STATISTICAL ANALYSIS}

Comparisons between patient groups for clinical and demographic variables were made using $t$ tests and $\chi^{2}$ tests. 
Table 1 Clinical and demographic details on patients with benign and non-benign multiple sclerosis (MS) in 1996 epidemiological study

\begin{tabular}{lll}
\hline Clinical/demographic details & Benign $M S$ & Non-benign MS \\
\hline No of patients & 36 & 145 \\
Women (n (\%)) & $29(80.6)$ & $98(67.6)$ \\
Men (n (\%)) & $7(19.4)$ & $47(32.4)$ \\
Age at assessment (mean (SD)) & $45.3(10.9)$ & $54.9(11.7)$ \\
Age at onset (mean (SD)) & $25.8(6.9)$ & $31.2(10.1)$ \\
Disease duration (mean (SD)) & $19.7(8.5)$ & $23.9(9.7)$ \\
Mean EDSS & 1.9 & 6.2 \\
Median EDSS & 2.0 & 6.0 \\
Pyramidal FS score $\geqslant 3(\mathrm{n}(\%))$ & $1(2.8)$ & $123(84.8)$ \\
In full employment (n (\%)) & $24(66.7)$ & $4(2.8)$ \\
In receipt of external support (n (\%)) & $10(27.8)$ & $140(96.6)$ \\
\hline
\end{tabular}

Table 2 Clinical and demographic details on patients with age at onset over 40 and under 40 in 1996 epidemiological study and in whom EDSS was calculated

\begin{tabular}{lll}
\hline Clinical/demographic details & Age of onset $>40$ & Age of onset $<40$ \\
\hline No of patients & 58 & 201 \\
Women (n (\%)) & $35(60.3)$ & $144(71.6)$ \\
Men (n (\%)) & $23(39.7)$ & $57(28.4)$ \\
Age at assessment (mean (SD)) & $60.8(9.6)$ & $46.3(12.3)$ \\
Age at onset (mean (SD)) & $46.2(5.9)$ & $27.1(6.7)$ \\
Disease duration (mean (SD)) & $14.6(8.9)$ & $19.4(11.5)$ \\
Mean EDSS & 5.7 & 4.7 \\
Median EDSS & 6.0 & 5.0 \\
Following benign course (n) & 1 & 35 \\
Following progressive course from onset (n (\%)) & $21(36.2)$ & $11(5.5)$ \\
\hline
\end{tabular}

Table 3 Clinical and demographic details in age groups under 40 at onset of multiple sclerosis (MS)

\begin{tabular}{llll}
\hline Clinical/demographic details & $\begin{array}{l}\text { Age of onset } \\
<20\end{array}$ & $\begin{array}{l}\text { Age of onset } \\
20-29\end{array}$ & $\begin{array}{l}\text { Age of onset } \\
30-39\end{array}$ \\
\hline No of patients & 29 & 98 & 74 \\
Women (n (\%)) & $24(82.8)$ & $68(69.4)$ & $52(70.3)$ \\
Men (n (\%)) & $5(17.2)$ & $30(30.6)$ & $22(29.7)$ \\
Age at assessment (mean (SD)) & $35.6(11.3)$ & $46.5(12.5)$ & $50.2(9.8)$ \\
Age at onset (mean (SD)) & $16.6(2.2)$ & $24.9(2.6)$ & $34.2(3.3)$ \\
Disease duration (mean (SD)) & $19.1(11.3)$ & $21.7(12.4)$ & $16.1(9.5)$ \\
Mean EDSS & 4.6 & 4.8 & 4.5 \\
Median EDSS & 5.0 & 5.5 & 5.0 \\
Following benign course (n (\%)) & $7(33.3)$ & $16(21.1)$ & $12(23.1)$ \\
Following progressive course from onset (n (\%)) & $1(3.4)$ & $5(5.1)$ & $7(9.5)$ \\
\hline
\end{tabular}

Ethical approval for the study was granted by the Queen's University of Belfast research ethics committee.

\section{Results}

BENIGN MULTIPLE SCLEROSIS GROUP 1996

Of 181 patients with an EDSS measured at least 10 years from the date of onset of their symptoms, $36(19.9 \%)$ had a benign course. The demographic details of these patients are shown in table 1 . There were considerably more women than men and the ratio (4.1:1) was greater than that identified in the overall epidemiological study for definite/probable disease $(2.3: 1)$ or all cases $(2.1: 1)$.

For mode of onset, the commonest symptoms were optic neuritis $12(33.3 \%)$, and sensory disturbance $12(33.3 \%)$. Brainstem or cerebellar disturbance at onset occurred in 10 cases $(27.8 \%)$ and motor symptoms presented at onset in six cases (16.7\%). Disability was low, as would be expected, with mean and median EDSS scores of 1.9 and 2.0 respectively. These low scores are reflected in the pyramidal FS scores, in which just one patient $(2.8 \%)$ had a score of 3 , whereas nine $(25 \%)$ scored 2, $15(41.7 \%)$ scored 1 , and $11(30.6 \%)$ scored 0. All 36 patients had exhibited a relapsing-remitting course from onset. For Poser classification of the clinical course, 18
(50\%) had CDMS, 12 (33.3) had CPMS, four $(11.1 \%)$ had LSDMS, and two $(5.6 \%)$ had LSPMS.

By contrast, there were 145 patients with an EDSS $>3.0$ at least 10 years after onset. The demographics of this group are also shown in table 1 . The mean age at onset was significantly higher than in the benign group $(\mathrm{p}<0.001)$. Symptoms at onset were sensory $46(31.7 \%)$; brainstem/cerebellar 38 (26.2\%); motor 35 $(24.1 \%)$; and optic neuritis $30(20.7 \%)$.

Compared with benign multiple sclerosis, $123 / 145$ patients $(84.8 \%$, ) had a pyramidal FS score $\geqslant 3$ ( $v$ benign multiple sclerosis, $\mathrm{p}<0.001)$. Just 26 patients $(17.9 \%)$ remained in the relapsing-remitting phase of the disease ( $v$ benign multiple sclerosis, $\mathrm{p}<0.001$ ), and 14 $(9.7 \%)$ had had a progressive course from onset ( $v$ benign multiple sclerosis, $\mathrm{p}=0.04$ ).

IMPACT OF AGE AT ONSET ON CLINICAL COURSE Given the significantly younger age at onset of the benign group, we sought to evaluate the association of this variable with subsequent clinical course in the whole group of 259 patients undergoing an EDSS assessment.

Firstly, drawing a line at 40 years of age, there were 58 patients with onset at this age or later, compared with 201 having an earlier onset. The clinical and demographic details on the two groups are given in table 2. Just one patient from the older than 40 group fulfilled the criteria for benign multiple sclerosis, 34 having had the disease for at least 10 years. In 55 patients the symptoms at onset could be established as motor 27 (49.1\%); sensory 16 (29.1\%); brainstem/cerebellar 8 (14.5\%); optic neuritis $4(7.3 \%)$.

All but one (97.2\%) of the 36 patients with benign multiple sclerosis were drawn from the group with onset under 40. Despite the significantly longer duration of disease in this group (19.4 v 14.7 years, $\mathrm{p}<0.001$ ), disability was less. Symptoms at onset were established in 200 as sensory $73(36.5 \%)$; brainstem/ cerebellar 55 (27.5\%); motor 45 (22.5\%); optic neuritis $41(20.5 \%)$.

To determine if the age at onset under 40 is important prognostically, the data for this group were further subdivided into those with onset between 30 and 39 years, 20 and 29 years, and under 20 years. The data for these groups appear in table 3. No clear pattern emerged. The mean and median EDSS scores in each of the groups were similar. Although the proportion of patients with benign multiple sclerosis was highest in the under 20 age group, the difference between this and the other two groups was not statistically significant. However the proportion of women was greatest in the under 20 group and the proportion of patients with progressive disease from onset increased through the three age groups.

FOLLOW UP OF THE BENIGN MULTIPLE SCLEROSIS GROUP 1986-7

The previous epidemiological study in 1986-7, in Coleraine, Ballymoney, and Moyle, identified 118 patients with definite, probable, and possible multiple sclerosis. Of these, 78 re- 
Table 4 Clinical and demographic details on patients benign and non-benign multiple sclerosis (MS) followed up from 1986-7 study

\begin{tabular}{lll}
\hline Clinical/demographic details & Benign $M S$ & Non-benign MS \\
\hline No of patients & 28 & 42 \\
Women (n (\%)) & $21(75.0)$ & $26(61.9)$ \\
Men (n (\%)) & $7(25.0)$ & $16(38.1)$ \\
Age at assessment (mean (SD)) & $57.0(12.6)$ & $52.5(12.3)$ \\
Age at onset (mean (SD)) & $27.7(9.0)$ & $32.8(9.5)$ \\
Disease duration (mean (SD)) & $29.4(9.7)$ & $19.8(8.3)$ \\
Mean EDSS (1987) & $4.6(2.3)$ & $6.2(4.1)$ \\
Median EDSS (1987) & $5.0(3.0)$ & $6.5(5.0)$ \\
Pyramidal FS score $\geqslant 3(\mathrm{n}(\%))$ & $18(64.3)$ & $34(81.0)$ \\
In progressive phase (n (\%)) & $15(53.6)$ & $34(81.0)$ \\
In full employment (n (\%)) & $6(21.4)$ & $5(11.9)$ \\
In receipt of external support (n (\%)) & $18(64.3)$ & $39(92.9)$ \\
\hline
\end{tabular}

mained alive and resident within the study area for 1996 . Of the 33 patients with benign multiple sclerosis identified in $1986-7,28$ were available for follow up, five patients having died in the intervening period. All of these had an EDSS assessment in 1996. Of the other 50 surviving patients with non-benign multiple sclerosis, 42 underwent a DSS assessment in 1987 and an EDSS in 1996. A comparison of the respective demographic data in the follow up groups is given in table 4 .

In 1986-7 the mean DSS of the benign group was 2.3 and the median was 3.0. By 1996, disability in the group had progressed to mean EDSS 4.6, median 5.0. The change in disability in this group over time is shown in the figure. In the first study, 26/28 patients were in the relapsing-remitting phase of the disease, two having had a slowly progressive course from onset. By 1996, 13 had moved from the relapsing-remitting to the secondary progressive phase. Just eight patients $(28.6 \%)$ continued to fulfil the criteria for benign multiple sclerosis, and it is of note that all of these patients had DSS scores $\leqslant 2.0$ in $1986-7$. The commonest symptoms at onset in this group were sensory $(10,35.7 \%)$ and brainstem/ cerebellar $(10,35.7 \%)$. Six patients $(21.4 \%)$ had optic neuritis initially and five $(17.9 \%)$ had motor disturbance.

Among the non-benign multiple sclerosis group in 1986-7 the mean and median DSS scores had been 4.1 and 5.0 respectively and by 1996 these had reached 6.2 and 6.5 respec-

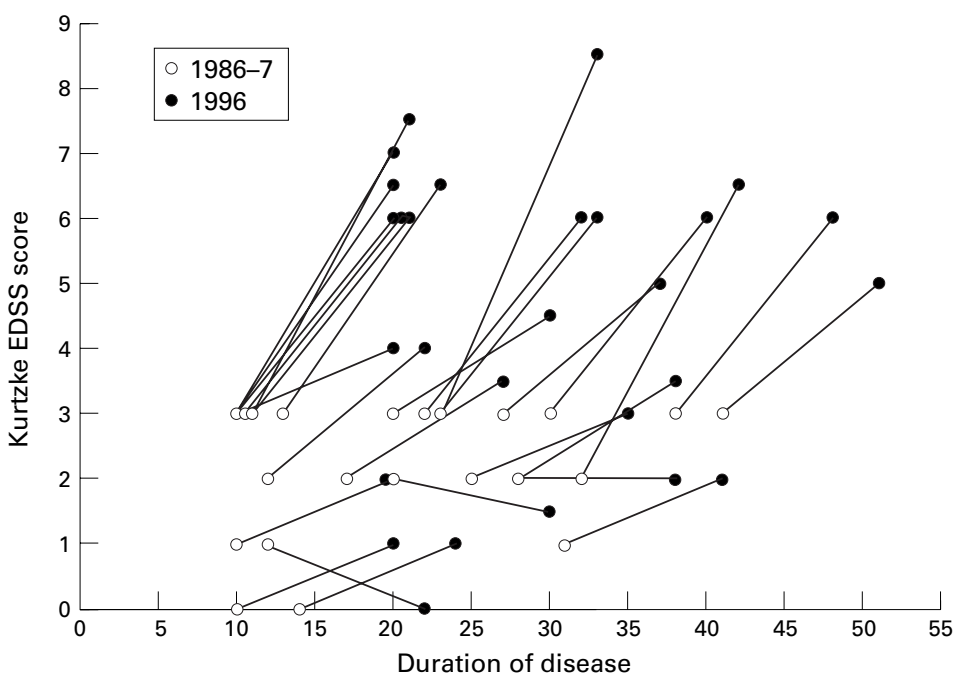

Change in disability in "benign multiple sclerosis" between 1986-7 and 1996 tively. Five of the group had progressive disease from onset and just eight of the remainder had not entered the secondary progressive phase. This group of eight had a relatively short duration of disease (mean 13.4 years) and none would have been eligible for inclusion in the benign category of 1986-7. The symptoms at onset were established in 40 of 42 patients as motor $11(27.5 \%)$; optic neuritis $11(27.5 \%)$; brainstem/cerebellar 10 (25\%); and sensory eight $(20 \%)$.

\section{Discussion}

This study and analysis sought to establish those features associated with a relatively favourable multiple sclerosis course in this population. A follow up of patients previously categorised as having benign multiple sclerosis has also been undertaken to evaluate if the initially favourable course is subsequently maintained. The study was population based with accurate case ascertainment.

From the first part of the study, incorporating the data from the 1996 survey only, the female:male ratio is greater in the benign than in the non-benign multiple sclerosis group (4.1:1 $v 2.1: 1)$. Although this did not reach significance because of the small numbers involved $(p=0.13)$, it does suggest that women are about twice as likely to follow a benign course as men (odds ratio (OR) $2.0,95 \%$ confidence interval $(95 \% \mathrm{Cl}) 0.8-5.4)$. This is in line with the results of others..$^{1820}$ The mean age at onset was also significantly less in the benign group, on average being 5.4 years younger than in the non-benign group.

No clear conclusions can be drawn from the symptoms at onset in the benign and nonbenign groups although optic neuritis and sensory disturbance were the commonest symptoms at onset in the benign group and this again has been a common experience. ${ }^{681418202330}$ The lesser incidence of motor symptoms at onset in the benign group at onset can be seen to impact favourably on long term pyramidal function with resultant benefits in terms of disability and handicap. Although the benign group was younger (45.3 $v 54.9$ ) and had a shorter mean disease duration (19.7 $v 23.9$ ), the differences with regard to employment $(66.7 \%$ v $2.8 \%$, $\mathrm{p}<0.001)$ and welfare support $(27.8 \% v 96.6 \%$, $\mathrm{p}<0.001)$ are still striking.

Age at onset over 40 was associated with a more disabling course. Whereas the older age of the older than 40 group at the time of assessment must be taken into account, even with a significantly shorter mean duration of disease $(p<0.001)$, mean and median EDSS scores for this group were still 1.0 higher than in those with onset of disease before 40 . The older than 40 group were significantly more likely to have motor disturbance at onset $(49.1 \%$ v $22.5 \%, \mathrm{p}<0.001$; OR $3.3,95 \% \mathrm{Cl}$ 1.7-6.5) whereas those under 40 were more likely to have optic neuritis at onset $(20.5 \% v$ $7.3 \%, \mathrm{p}=0.02$; OR $3.3,95 \% \mathrm{Cl} 1.1-11.4)$. Those older than 40 at onset were also more likely to have progressive disease from onset $(36.2 \%$ v $5.5 \%, \mathrm{p}<0.001$; OR $9.8,95 \% \mathrm{Cl}$ 4.1-23.9). 
In the age groups under 40 the distinctions were less clear. However, those with onset under 20 were more likely to have a benign course and greater female predominance and less likely to have a progressive course from onset. Overall these data support the findings of others indicating that onset after age 40 is generally associated with an unfavourable course. ${ }^{12} 141823$

The follow up of patients with benign multiple sclerosis identified in 1986-7 emphasises some of the characteristics seen in the benign multiple sclerosis group from the more recent study. The female predominance was greater (ratio 3:1 $v 1.6: 1, \mathrm{p}=0.25$ ) and the age at onset was earlier than in a non-benign group also available for follow up from 1986-7 (27.7 v 32.8 years, $\mathrm{p}=0.02$ ). Sensory symptoms were again common in the benign group, with motor symptoms rather less so, and the clinical course was characteristically relapsing-remitting from onset. Also, despite an older age at follow up and longer duration of disease (29.4 $v 19.8$, $\mathrm{p}<0.001)$, mean and median EDSS scores remained significantly lower in the benign group (4.6 and 5.0 respectively $v 6.2$ and 6.5). The proportions of patients in the progressive phase $(53.6 \%$ v $81.0 \%, \mathrm{p}=0.01)$, in full employment $(21.4 \% v 11.9 \%, \mathrm{p}=0.28)$ and in receipt of external support $(64.3 \% \vee 92.9 \%$, $\mathrm{p}=0.003$ ) all remained in favour of the benign group.

This part of the study does, however, serve as a reminder that the term "benign" is relative and often temporary. It is noteworthy that $50 \%$ of those in the benign group who were in the relapsing-remitting phase in 1986-7 had entered the secondary progressive phase 10 years later. Also, the rate of deterioration was actually similar in both benign and non-benign groups, the mean EDSS progressing from 2.3 to 4.6 in the benign multiple sclerosis group and from 4.1 to 6.2 in the non-benign group. This indicates that although the EDSS/DSS does provide a useful indicator of prognosis in multiple sclerosis, apparently benign disease often becomes disabling. The overall data confirm the previous experience in the studies of McAlpine, in which a 5 year follow up study showed that 12 of 78 patients with benign multiple sclerosis had become disabled and three had died. ${ }^{631}$

Some caution is required in the interpretation of the data, not least because of the relatively few patients involved. A further factor is that the loss of patients from the 1986-7 study, particularly due to death or transfer to centres for long term care, may underrepresent the degree of disability in both the benign and especially the non-benign follow up groups. Another problem is the categorisation of patients with mild or minimal disability but less than 10 years duration who do not yet fulfil the criteria for benign multiple sclerosis but who may well subsequently do so.

In conclusion, the "loss" of patients from the benign category over time suggests that a progression index rather than arbitrary disease duration/EDSS cut off may be a more relevant criterion for defining this aspect of the disease.
Each of the authors contributed equally to this work.

GVMcD has been a Research Fellow at the Royal Victoria Hospital in Belfast and was also supported by the charity Action multiple sclerosis. We acknowledge the contribution of $\mathrm{Dr}$ Frank Kee to the original study in 1986-7 and the the assistance of all those involved in the case ascertainment for both studies.

1 Sadovnick AD, Armstrong H, Rice GPA, et al. A population-based study of multiple sclerosis in twins: update. Ann Neurol 1993;33:281-5.

2 Gilbert JJ, Sadler M. Unsuspected multiple sclerosis. Arch Neurol 1983;40:533-6.

3 Herndon RM, Rudick RA. Multiple sclerosis: the spectrum of severity. Arch Neurol 1983;40:531-2.

4 Poser S, Wikström J, Bauer HJ. Clinical data and the identification of special forms of multiple sclerosis in 1271 cases
studied with a standardized documentation system. $f \mathrm{Neu}$ studied with a standardize
rol Sci 1979;40:159-68.

5 Hutchinson M. Disability due to multiple sclerosis: a community-based study of an Irish county. Ir Med F 1986; 79:48-50.

6 McAlpine D. The benign form of multiple sclerosis. A study based on 241 cases seen within 3 years of onset and followed up until the tenth year or more of the disease. Brain 1961;84:186-203.

7 Bauer HJ, Firnhaber W, Winkler W. Prognostic criteria in multiple sclerosis. Ann N Y Acad Sci 1965;122:542-51.

8 Riser M, Geraud J, Rascol A, et al. L'évolution de la sclérose en plaques (étude de 203 observations series au dela de 10 ans). Rev Neurol 1971;124:479-84.

9 Kurtzke JF, Beebe GW, Nagler B, et al. Studies on the natural history of multiple sclerosis VIII. Early prognostic features of the later course of the illness. 7 Chron Dis 1977; 30:819-30.

10 Thompson AJ, Hutchinson M, Brazil J, et al. A clinical and laboratory study of benign multiple sclerosis. $Q f M$ 1986;225:69-80

11 Lublin FD, Reingold SC. Defining the clinical course of multiple sclerosis: results of an international survey. Neurology 1996:46:907-11.

12 Leibowitz U, Alter M. Clinical factors associated with increased disability in multiple sclerosis. Acta Neurol Scand 1970;46:53-70.

13 Confavreux C, Aimard G, Devic M. Course and prognosis of multiple sclerosis assessed by the computerised data processing of 349 patients. Brain 1980;103:281-300.

14 Poser S, Bauer HJ, Poser W. Prognosis of multiple sclerosis. Results from an epidemiological area in Germany. Acta Neurol Scand 1982;65:347-54.

15 Verjans E, Theys P, Delmotte P, et al. Clinical parameters and intrathecal IgG synthesis as prognostic features in multiple sclerosis. Part I. I Neurol 1983;229:155-65.

16 Patzold U, Pocklington PR. Course of multiple sclerosis. First results of a prospective study carried out of $102 \mathrm{MS}$ patients from 1976-80. Acta Neurol Scand 1982;65:24866.

17 Fog T, Linnemann F. The course of multiple sclerosis in 73 cases with computer designed curves. Acta Neurol Scand 1970;46(suppl 47):1-175.

18 Weinshenker BG, Rice GPA, Noseworthy JH, et al. The natural history of multiple sclerosis: a geographically based natural history of multiple sclerosis: a geographically based
study. 3. Multivariate analysis of predictive factors and study. 3. Multivariate analysis of predictive
models of outcome. Brain 1991;114:1045-56.

19 Runmarker B, Andersen O. Prognostic factors in a multiple sclerosis incidence cohort with 25 years of follow-up. Brain 1993;116:117-34

20 McAlpine D, Compston N. Some aspects of the natural history of disseminated sclerosis. $Q f M$ 1952;82:135-67.

21 Panelius M. Studies on epidemiological, clinical and etiological aspects of multiple sclerosis. Acta Neurol Scand 1969;45(suppl 39): 1-82.

22 Detels R, Clark VA, Valdiviezo NL, et al. Factors associated with a rapid course of multiple sclerosis. Arch Neurol 1982; 39:337-41.

23 Visscher BR, Liu K-S, Clark VA, et al. Onset symptoms as predictors of mortality and disability in multiple sclerosis. Acta Neurol Scand 1984;70:321-8.

$24 \mathrm{McD}$ onnell GV, Hawkins SA. An epidemiologic study of multiple sclerosis in Northern Ireland. Neurology 1998;50 423-8.

25 Poser CM, Paty DW, Scheinberg L, et al. New diagnostic criteria for multiple sclerosis: guidelines for research protocols. Ann Neurol 1983;13:227-31.

26 Paty DW, Ebers GC. Clinical features. In: Paty DW, Ebers GC, eds. Multiple sclerosis. Philadelphia: FA Davis, 1998: $151-2$.

27 O'Riordan JI, Thompson AJ, Kingsley DPE, et al. The prognostic value of brain MRI in clinically isolated syndromes of the central nervous system. A 10 year follow up. Brain 1998;121:495-504

28 Kurtzke JF. A proposal for a uniform minimal record of disability in multiple sclerosis. Acta Neurol Scand 1981; 64(suppl 87):110-29.

29 Mellerup E, Fog T, Raun N, et al. The socio-economic scale. Acta Neurol Scand 1981;64(suppl 87):130-8.

30 Sanders EACM, Bollen ELEM, van der Velde EA. Presenting signs and symptoms in multiple sclerosis. Acta Neurol ing signs and symptom

31 McAlpine $\mathrm{D}$. The benign form of multiple sclerosis: results of a long-term study. $B M \mathcal{F} 1964 ; 2: 1029-32$. 